\title{
Computational and Visual Electromagnetics Using an Integrated Programming Language for Undergraduate Engineering Students
}

\author{
Junwei Lu, Member, IEEE, and David V. Thiel, Senior Member, IEEE
}

\begin{abstract}
Numerical modeling is now an essential part of undergraduate engineering education. This paper reviews the content and delivery of a course for undergraduate engineers, in computational and visual electromagnetics using the integrated programming language, Matlab. A number of numerical methods are discussed including FDM, FEM, MOM and BEM during class. Matlab is then used to implement these methods in a series of workshops, laboratories exercises and assignments.
\end{abstract}

Index Terms-Computational and visual electromagnetics, computational science and engineering, engineering education, integrated programming language.

\section{INTRODUCTION}

$\mathbf{C}$ OMPUTATIONAL and visual electromagnetics (CVEM) software packages are now widely used in science and engineering practice. It is very important to introduce the concept of computational engineering to undergraduate study in the electromagnetics, as electromagnetic simulation has moved from analysis to design in engineering practice. Conventional education in electromagnetics does not use computation and visualization techniques to help student learning. The very complicated analytical solutions, formulas and derivations often lead students to fail the electromagnetic subjects. Also conventional analytical methods can not solve many real and practical engineering problems. Therefore, the simplified analytical solutions are often useless in engineering design and analysis. To solve such problems, a new educational program in CVEM has been introduced into undergraduate courses [1], [2]. This paper reviews the content and delivery of a new subject in computational science and engineering for CVEM using an integrated programming language for undergraduates in the School of Microelectronic Engineering. Through several years teaching practice and feedback from students, we have achieved significant success. The students with such skill have been highly recognized by employers in industry.

\section{EDUCATIONAL RATIONALE OF CVEM}

Computational science and engineering (CSE) is a young and a relatively new and rapidly evolving field. O'Leary [3] defined CSE as: "CSE is an interdisciplinary approach to the solution

Manuscript received October 25, 1999.

The authors are with the School of Microelectronic Engineering, Faculty of Engineering, Griffith University, Nathan, Qld 4111, Australia (e-mail: \{J.Lu; D.Thiel\}@me.gu.edu.au).

Publisher Item Identifier S 0018-9464(00)06392-5.

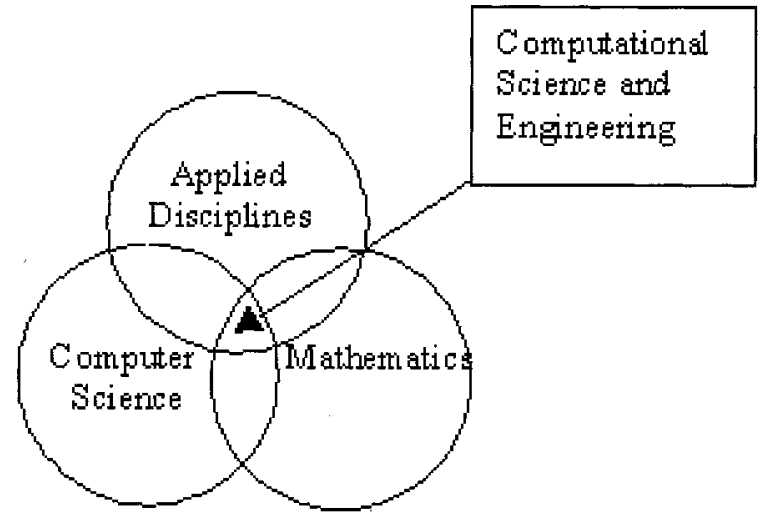

Fig. 1. The definition of computational science and engineering.

of problems in the natural science and technology, drawing on the tools of a science or engineering discipline plus those of computer science plus those of mathematics." The definition of CSE can be also described by Fig. 1. Therefore, training in computational science and engineering aims to train scientists and engineers in core discipline areas described as follows.

\section{A. Educational Strategy}

There are three main elements we consider essential in teaching the subject of computational electromagnetics:

1) Students must be involved in developing the algorithms without becoming bogged down in complex programming for such tasks as matrix inversion and data visualization.

2) Students must learn to verify their code using models which have analytical solutions. Their solutions must not only be verified by 2D or 3D plots, (e.g. shape of curves), but also by quantitative numerical comparison between the analytical solution and their modeled solution. This is essential practice for both student codes and commercial packages.

3) Excessive assessment of simple or trivial exercises is not desirable. Individualized projects following nonassessed, non- compulsory, learning workshops have generated considerable student enthusiasm for the subject.

\section{B. Natural Consequences of These Strategies}

With these three strategies in place, a number of desirable outcomes have been achieved. These outcomes include: 
1) Students dedicated considerable time and research to their projects.

2) Students returned to their theoretical textbooks to seek out and understand the analytical solutions and to interpret complex equations and the nature of EM fields.

3) Individualized assignments were not difficult to set for up to 35 students, and marking was not difficult because the code verification was highly valued in the over-all making criteria.

4) The great ability of graduates from the course to tackle very complex problems to obtain practical useful results is highly valued by employers. Employment in the fields of engineering software development, electromagnetics and general electrical and electronic engineering was greatly enhanced.

\section{Integrated Programming Languages}

Integrated programming languages (IPL) are also called interactive programming languages that combine attractive, easily produced graphics with powerful support for mathematical modeling. Typically, these languages support both a high-level programming languages and packages for symbolic or algebraic manipulation, matrix operations, and visualization. Examples of such products include Macsyma, Maple, Mathematica, Matlab and Mathcad. The interactive languages have become popular at universities and industries [4], [5]. Particularly, we use MATLAB based integrated programming language for this subject. It must be emphasized that all students in the School of Microelectronic Engineering take at least one subject which is MATLAB based, and with most students MATLAB is their preferred integrated programming language because of the ease of use. The requirement in the subject for additional learning of programming techniques is quite small, especially for the visualization section in the program. For instance, after the electric static field is calculated by using any numeric methods, such as FDM or FEM, we want to plot 2-D electric potential $V(x, y)$ and electric field ( $E x$ and $E y$ ) in the same figure, a few in-built functions are required for the program; for example:

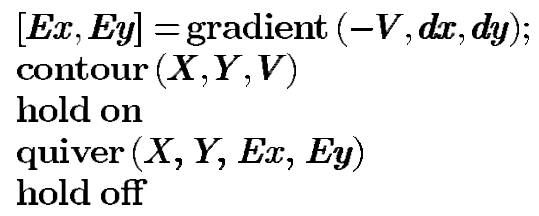

We use the definition of the gradient derivative to describe the relationship between the electric potential $V(x, y)$ and the electric field $\vec{E}(x, y)$ as

$$
\vec{E}=-\nabla V
$$

The direction of the electric field will be perpendicular to the lines of constant potential, and the minus sign means that it will point "downhill." Therefore the gradient is used to compute the electric field from the electric potential $V$ which is a matrix of the values of the potential at specific grid points, and $d x, d y$ are the spacings of the grid in the $x$ and $y$ directions, respectively. The quiver is used to present the result on a graph. $X, Y$ are the matrices of the grid points and $E x$ and $E y$ are the $x, y$ components of tiny arrows that are drawn at each grid point. The function quiver is used most often in conjunction with a contour plot to indicate not only the lines of constant contour, but also the direction of steepest ascent.

For 3-D potential contour plot, we often use following built-in functions to visualize the results as a surface plot.

$\operatorname{mesh}(X, Y, V)$

The animation function is very useful to present wave propagation in both time domain or frequency domain simulation. We can create animated sequences with MATLAB in two different ways; movies and erase modes. For the movie mode, we use moviein to initialize memory for a matrix large enough to hold the specified number of frames based on the size of the current axis, and getframe to generate each movie frame, which it returns as a column vector, we can then build into a movie matrix, and finally use movie to run the movie at the specified number of times. The example program for electromagnetic wave propagation can be written as follows:

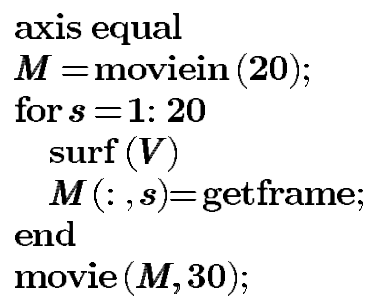

\section{Course Structure}

The CVEM course called "Numerical Techniques in Electromagnetics" has been successfully taught at School of Microelectronic Engineering, Griffith University in Australia since 1992. Many students graduated from this school with this computational EM skill have been highly recognized by their employers in industry. Because many commercially available software packages are widely used in industry such as: MOM based Ensemble, MININEC and NEC, and IE3D, FEM based HFSS and MAXWELL 3D, FD-TD based XFD-TD and FIDELITY, BEM based ELECTRO and OERSTED, and so fourth. All those Engineering analysis and design packages require intensive training time. If the graduates do not have any CVEM background it will take them few months to learn how to use those software properly. But for our students, it only takes them few weeks to get familiar with those software packages. The details of course structure and course content are presented as follows:

1) Basic Numerical Analysis with MATLAB: In this section, the fundamental numerical methods such as matrices, matrix inversion, solving linear system equations using direct methods and iterative methods, numerical differentiation and integration, ordinary differential equations (ODE's) and partial differential equations (PDE's) are introduced, and implemented using MATLAB based integrated programming language [2].

2) Finite Difference Method (FDM) in EM Fields: FDM is one of the major sections in this course. Finite difference equations for Laplace's, Poisson's and wave equations, with 
associated boundary conditions and initial conditions are taught. Several application problems such as 2-D electro-static field and wave propagation problems are solved in the classroom. FD-TD for 2-D and 3-D time domain problems is also discussed in this section.

3) Finite Element Method (FEM) in EM Fields: FEM is another major section in this course. In the FEM theory section, energy functional minimization and Galerkin's method, mesh generation, and boundary conditions are introduced. Due to the complexity of FEM theory compared with FDM, only 2-D FEM for electric and magnetic static fields and time harmonic field problems (FE-FD) and time domain problems (FE-TD) as the application problems are solved in the classroom.

4) Method of Moment (MOM) in EM Field: This section mainly discusses the fundamental theory of MOM and its application in the wire antenna and arrays. The MOM theory for the calculation of scattering problems from wire antennas and arrays is discussed in the classroom.

5) Other Numerical Methods in EM Field Computation: Several other numerical methods such as Boundary Element Method (BEM) and Transmission Line Method in EM are also introduced in this section. The application of BEM in the unbounded EM structure problems and TLM method for EM problems are discussed in this section.

6) High Performance Numerical Computation and Visualization: The basic concept of high performance parallel computing and visualization are briefly introduced in this section. The importance of using parallel computing and visualization techniques and its applications in EM are also discussed in the classroom.

\section{Teaching Methods And Assessment}

The subject is based on the electromagnetic theory and electrical and electronic engineering background of students in their previous year's study. The course is assessed by three assignments worth $60 \%$ and a final examination worth $40 \%$.

\section{A. Teaching Methods}

1) Lecture: 28 hours of lectures cover basic numerical techniques (6 hours), error control and validation of results ( 2 hours), high performance computation and visualization (4 hours), and major commonly used numerical methods for electromagnetic analysis and design, such as FDM and FD-TD (4 hours), FEM (4 hours), MOM (4 hours), BEM (4 hours) and other numerical methods (4 hours).

2) Workshop: $14 \times 4$ hours workshops are designed for computing exercise and some commercial package use, where mini lectures are also carried out to help students using the packages and write MATLAB programs and completing their assignments. A demonstrator is also involved in the workshop teaching. Six workshop sheets are prepared for students in the workshop session:

1) MATLAB based numerical computation and visualization,

2) Solving matrix equations using MATLAB

3) Solving 2-D electric field problems using FDM

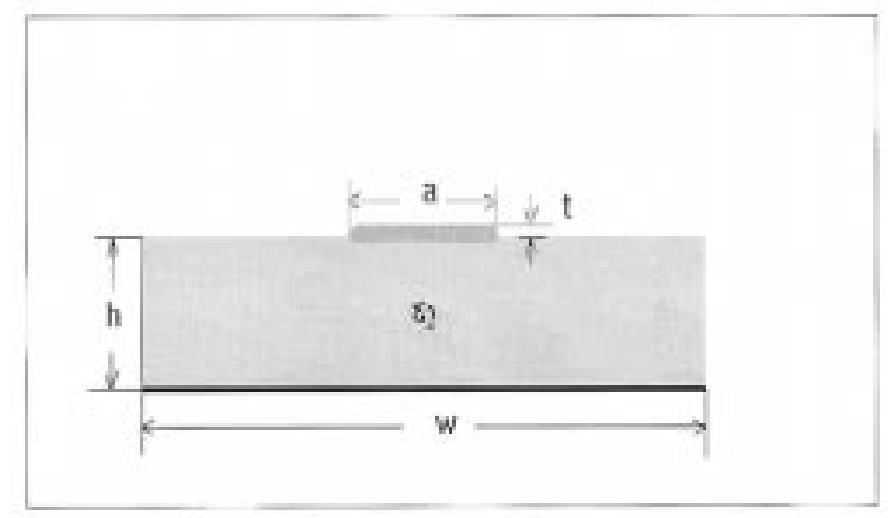

Fig. 2. One of FDM and FEM assignments, electric field analysis.

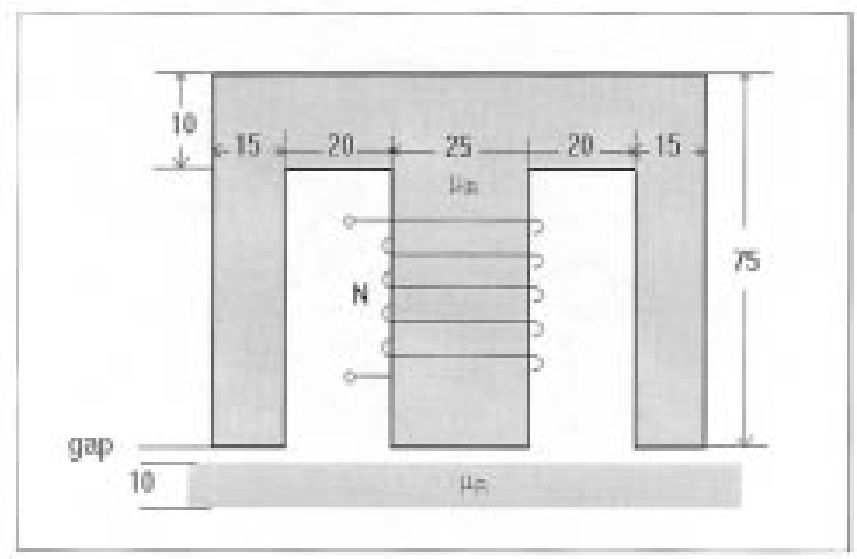

Fig. 3. One of FEM assignments, magnetic field simulations.

4) Solving 2-D electric and magnetic field problems using FEM

5) Solving electric and time harmonic magnetic problems using BEM based software packages, Electro and Oersted [6].

6) Wire antenna design and simulation using MOM based software packages, MININEC and NEC for solution [7] and MATLAB for post presentation.

\section{B. Assignments}

Three assignments are designed for students, where FDM, FEM and MOM techniques are required to be used for solving the various application problems.

In Assignment I (FDM), students select a 2-D electrostatic field problem from a number of application problems using the FDM techniques, such as one of the application problems, a microstrip transmission line as shown in Fig. 2.

In Assignment II (FEM), students can also select a problem from a large number of application problems using the FEM techniques. For instance, 2-D electrostatic field, magnetostatic field problems, and time harmonic field problems using reduced analysis models are options. Fig. 3 shows one of the application problems, a magnetic field simulation.

In assignment I and II, students should consider the problem definition, material properties involved in the analysis region, 


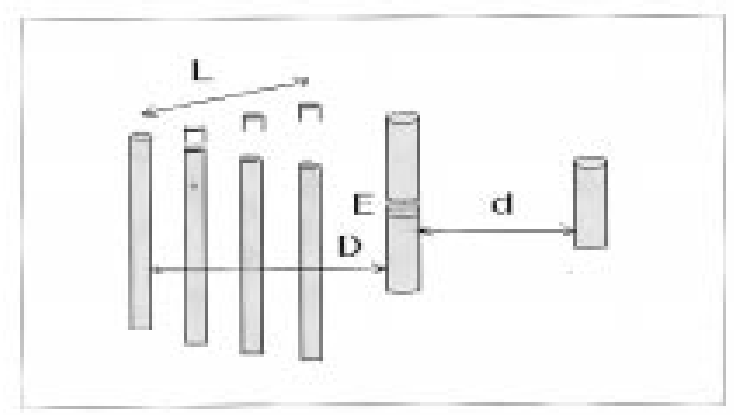

Fig. 4. Typical MOM assignments, corner reflector antenna simulation where $E$ is the excitation point.

structure symmetry, material properties, boundary conditions, method of filling matrices, matrix solving, three basic steps of computation procedures (pre-process, calculation and post-process), MATLAB program development, verification and presentation of solutions.

Assignment III requires students to solve a thin wire antenna array problem using MOM based on the MININEC and NEC packages [7], and MATLAB for data visualization and graphic plot. A large number of practical wire antenna and array problems are available for students to choose. Fig. 4 shows one of application problem in antenna design and analysis. In each problem, students should consider the problem definition, antenna structure and location, post-process using MATLAB visualization program, verification and presentation of solutions.

\section{Examination}

Examination time is set up as 3 hours, it covers 1) matrix solving using direct method and indirect method, 2) comparison and discussion of four most popular numerical methods used by software industries, such as FDM, FEM, MOM and BEM, 3) programming flow chart for FEM and BEM, 4) error control and validation of results, 5) high performance computation and visualization.

\section{CONCLUSIONS}

Computational methods are now an important part of engineering education. Just as in the 1980's basic mathematics and science was augmented by instruction in computer programming, the next 10 years will see computational methods and visualization added into engineering education, either directly through a subject like the one described in this paper, or as an adjunct tool used in many different subjects. The next generation of PC's is so powerful that very large modeling problems can be solved, and graduate engineers can employ this computation power to advantage. Some educators might be tempted to argue that the use of commercially available packages is self-explanatory and no formal education is required. It is our view that blind faith in a package is very dangerous, and a specific course can and must explore the limitations of the method and techniques of verification of the solutions for the particular class of problem of interest at the time.

\section{ACKNOWLEDGMENT}

The authors wish to thank the demonstrators: D. Gray, S. Saario and S. Smith for their contribution during the teaching practice and workshops.

\section{REFERENCES}

[1] D. V. Thiel and J. Lu, "Numerical techniques in electromagnetics and communications-A PC based third year undergraduate subject for microelectronic engineering," in IEEE AP-S International Symposium and URSI Radio Science Meeting, USA, June 1994, pp. 111-114.

[2] J. Lu, D. Thiel, and S. Saario, "High performance numeric computation and visualization of electromagnetics using MATLAB," in The Seventh Biennial IEEE Conference on Electromagnetic Field Computation, IEEE CEFC'96, June 1996, p. 275.

[3] D. P. O'Leary, "Teamwork: Computational science and applied mathematics," IEEE Computational Science \& Engineering, pp. 13-18, April-June 1997.

[4] A. Biran and M. Breiner, MATLAB for Engineers: Addison-Wesley, 1995.

[5] L. K. Kale, "Programming languages for CSE: The state of the art," IEEE Computational Science \& Engineering, pp. 18-26, April-June 1998.

[6] IES, "Electro and Oersted," Integrated Engineering Software Inc., 1992.

[7] G. J. Burke and A. J. Poggio, NEEDS 2.0: NEC-81 Ver 2.2 and MININEC Ver 3.0, Naval Ocean Systems Center, 1986. 